Background and objectives During inflammation and tissue damage, pathogens and dying cells are ingested by different phagocytes such as macrophages and dendritic cells. Both the nature and the activation state of the respective phagocyte determine the resulting immune response, which ranges from specific immunity to tolerance. In this context, the coordinated removal of apoptotic cells is essential to prevent autoimmunity and imposes a special task on the innate immune system.

Materials and methods The authors both studied the removal of apoptotic thymocytes in vitro and the resulting immunological consequences in the pristine model of murine lupus in vivo.

Results During peritonitis the removal of apoptotic cells is performed by a distinct population of alternatively activated and 12/15-LO-expressing resident macrophages (resM $\Phi$ ). In the absence of $12 / 15-\mathrm{LO}$, this pattern was deranged and inflammatory macrophages (infM $\Phi$ ) started to engulf apoptotic cells.

The authors hypothesised a 12/15-LO-dependent block of phagocytosis in infM $\Phi$ and consequently identified 12/15$\mathrm{LO}$-derived oxidation products of phosphatidylethanolamine $(\mathrm{OxPE})$ as the corresponding mediators, which are generated by resM $\Phi$ to selectively block ingestion of apoptotic cells by infM $\Phi$.

In contrast, phagocytosis of bacteria, micropinocytosis or the ingestion of apoptotic cells by resM $\Phi$ was unaffected. $\mathrm{OxPE}$, which is exposed on the outer membrane of resM $\Phi$ in a 12/15-LO-dependent manner, bound to soluble milk fat globule-epidermal growth factor 8 protein and consequently blocked the major pathway responsible for the uptake of apoptotic cells into infM $\Phi$. In consistence, the authors observed a break in self-tolerance in aged 12/15-LO-deficient mice including spontaneous production of autoantibodies and occurrence of glomerulonephritis, which both exacerbated after apoptotic challenge in the pristane-induced model of experimental murine lupus.

Conclusions This data point towards a so far unrecognised role for lipid oxidation during the maintenance of self-tolerance and identify a mechanism, which orchestrates the celland context-specific uptake of antigens by different subsets of phagocytes, imposing a new paradigm in their understanding of the clearance of apoptotic cells.

\title{
A95 12/15-LIPOXYGENASE ORCHESTRATES THE CLEARANCE OF APOPTOTIC CELLS AND MAINTAINS IMMUNOLOGICAL TOLERANCE
}

Stefan Uderhardt, ${ }^{1}$ Martin Herrmann, ${ }^{1}$ Olga Oskolikova, ${ }^{2}$ Susanne Aschermann, ${ }^{3}$ Benjamin Frey, ${ }^{1}$ Reinhard Voll, ${ }^{1}$ Falk Nimmerjahn, ${ }^{3}$ Valery N Bochkov, ${ }^{2}$ Georg Schett, ${ }^{1}$ Gerhard Krönke ${ }^{1}$ Department of Internal Medicine 3, University of ErlangenNuremberg, Erlangen, Germany; ${ }^{2}$ Department of Vascular Biology, University of Vienna, Vienna, Austria; ${ }^{3}$ Department of Genetics, University of Erlangen-Nuremburg, Erlangen, Germany 